\title{
QUALIDADE DE PRODUTOS MINIMAMENTE PROCESSADOS E COMERCIALIZADOS EM GÔNDOLAS DE SUPERMERCADOS NAS CIDADES DE LAVRAS - MG, BRASÍLIA - DF E SÃO PAULO - SP
}

\author{
Quality of fresh-cut produce commercialized on supermarket shelves in the cities \\ of Lavras-MG, Brasília-DF, and São Paulo-SP
}

\author{
Nélio Ranieli Ferreira de Paula1, Eduardo Valério de Barros Vilas Boas², Luiz José Rodrigues ${ }^{3}$, \\ Rosilane Aparecida Carvalho ${ }^{4}$, Roberta Hilsdorf Piccoli ${ }^{5}$
}

\begin{abstract}
RESUMO
A produção de frutos e hortaliças minimamente processados mostrou crescimento relevante nos últimos anos, em razão de acentuadas mudanças no estilo de vida do consumidor, como redução das famílias, busca de conveniência e conscientização da necessidade de uma dieta alimentar saudável e que atenda às exigências de segurança alimentar. Objetivou-se, neste trabalho, caracterizar a qualidade química, físico-química e microbiológica dos produtos minimamente processados, coletados em gôndolas de supermercados em Lavras - MG, Brasília - DF e São Paulo - SP para orientar seus fornecedores e consumidores quanto à necessidade de maior controle de qualidade, devido aos riscos de contaminação de matérias-primas por microrganismos patogênicos e deteriorantes. 144 amostras foram coletadas e submetidas às determinações de $\mathrm{pH}$, acidez titulável e sólidos solúveis e avaliações microbiológicas (coliformes a $45^{\circ} \mathrm{C}$, Escherichia coli e Salmonella sp.). Os resultados mostraram variações significativas nos valores de $\mathrm{pH}$ e foi observada diminuição de acidez e sólidos solúveis, durante o armazenamento. Foi detectada contaminação por coliformes a $45^{\circ} \mathrm{C}$ sendo que, em $50 \%$ dessa presença, houve isolamentos de Escherichia coli, evidenciando contaminação oriunda de matéria-prima inadequadamente higienizada ou por sua presença nos manipuladores. Contudo, não foi detectada contaminação por Salmonella sp.. Os resultados obtidos indicaram a necessidade de implementação de Boas Práticas de Fabricação (BPF), no controle de qualidade desses produtos.
\end{abstract}

Termos para indexação: Microbiologia, qualidade, minimamente processados.

\section{ABSTRACT}

The production of fresh-cut fruits and vegetables has shown an outstanding growth in the latest years, due to the remarkable changes in the consumers' life style, such as family reduction, search for convenience and increased awareness of a wholesome food diet which meets the requirements of food safety. The aim of this work was to characterize the physicochemical and microbiological quality of fresh-cut produce collected from shelves of supermarkets in Lavras, Brasília and São Paulo as so to guide their suppliers and consumers for the need for an increased quality control due to the risks of contamination of raw material by pathogenic and decaycausing microorganisms. 144 samples were submitted to $\mathrm{pH}$, titratable acidity, soluble solid analyses and microbiological analyses (coliforms at $45^{\circ} \mathrm{C}$, Salmonella sp. and Escherichia coli). The results showed significant alterations in pH values. A decrease in acidity and soluble solids during storage was found. Contamination by coliforms at $45^{\circ} \mathrm{C}$ during manufacture was verified in $50 \%$ of the presence verified. We have isolated Escherichia coli, which evidences contamination coming from inadequately cleaned raw material or by their presence on the handlers. However, no contamination by Salmonella sp. was detected. Our results indicate the need of the implementation of the Good Handling Practices for the quality control of these produce.

Index terms: Microbiology, quality, fresh-cut produce.

(Recebido em 20 de outubro de 2005 e aprovado em 19 de março de 2008)

\section{INTRODUÇÃO}

O aumento do consumo de frutas e hortaliças, tanto fresca como parcialmente processadas, está associado à busca por conveniência na vida atual o que conduziu à produção de amplo número de produtos, que são oferecidos para consumo imediato (BEUCHAT, 2002). A agroindústria de alimentos processados prontos para o consumo tem se esforçado para preparar, manusear e entregar um produto fresco, saudável, seguro e conveniente para o consumidor (VANETTI, 2004).

\footnotetext{
'Engenheiro Agrônomo, Mestre em Ciência dos Alimentos - Departamento de Vigilância Sanitária de Lavras - Rua Zequinha Vilela, 142 , apto 101 - 37200 000 - Lavras, MG - nelioraniel@yahoo.com.br

'Engenheiro Agrônomo, Ph.D., Professor - Departamento de Ciência dos Alimentos/DCA - Universidade Federal de Lavras/UFLA - Cx. P. 3037 37200-000 - Lavras, MG - evbvboas@ufla.br

${ }^{3}$ Químico, Mestre em Ciência dos Alimentos - Departamento de Ciência dos Alimentos/DCA - Universidade Federal de Lavras/UFLA - Cx. P. 3037 37200-000 - Lavras, MG - rodrigues.lui3@uol.com.br

${ }^{4}$ Bióloga - Departamento de Ciência dos Alimentos/DCA - Universidade Federal de Lavras/UFLA - Cx. P. 3037 - $37200-000$ - Lavras, MG rosilane_aparecida1976@yahoo.com.br

${ }^{5}$ Engenheira de Alimentos, Doutora, Professora - Departamento de Ciência dos Alimentos/DCA - Universidade Federal de Lavras/UFLA - Cx. P. 3037 37200-000 - Lavras, MG - rhpiccoli@ufla.br
} 
Segundo a International Fresh-Cut Producers Association (IFPA, 2005), produtos minimamente processados são definidos como qualquer fruta ou hortaliça, ou ainda qualquer combinação delas, que foi alterada fisicamente a partir de sua forma original, embora mantenha o seu estado fresco. Independente do tipo, ele é selecionado, lavado, descascado e cortado, resultando num produto $100 \%$ aproveitável que, posteriormente, é embalado ou pré-embalado.

O manuseio excessivo durante o descasque, o fracionamento, a lavagem e as condições de aeração e embalagem aumentam os riscos da presença de microrganismos patogênicos transmissores de doenças ao consumidor (FANTUSI et al., 2004).

A qualidade e a segurança dos alimentos minimamente processados estão intimamente relacionados à metodologia de processamento, qualidade da matériaprima, uso de embalagens apropriadas e às condições adequadas de armazenamento (CANTWELL \& SUSLOW, 2002). Dados recentes registraram que, simultaneamente, ao aumento do consumo de frutas e hortaliças frescas, foi também verificada tendência de aumento do envolvimento desses produtos, em surtos de infecções alimentares (BEUCHAT, 2002; ROSA \& CARVALHO, 2000).

Apesar de todo o crescimento verificado no setor de minimamente processados no país, nos últimos anos, é crescente o relato de doenças infecciosas associadas ao consumo de frutas e hortaliças minimamente processadas, o que tem despertado o interesse de agências regulatórias, como a ANVISA (Agência Nacional de Vigilância Sanitária), e institutos de defesa dos direitos dos consumidores, como é o caso do IDEC (Instituto Brasileiro de Defesa do Consumidor) (ANDRADE et al., 2004).

No processamento mínimo, as barreiras para eliminação de microrganismos são poucas, constituindose as chamadas tecnologias de barreiras ou obstáculos, que incluem, principalmente, a lavagem, o uso de sanificantes, as embalagens em atmosfera modificada e a refrigeração (BERBARI et al., 2001).

Após serem processados, os produtos devem apresentar atributos de qualidade, como o frescor, aroma, cor e sabor mantendo o máximo de suas características nutritivas e sensoriais. Produtos minimamente processados são altamente perecíveis devido à exposição de seus tecidos internos, causando aceleração no seu metabolismo em decorrência da referida alteração física (CHITARRA \& CHITARRA, 2005). A combinação de tecido injuriado e aceleração no metabolismo contribuem grandemente para a perda de qualidade do produto, afetando, conseqüentemente, sua vida de prateleira (DELIZA, 2000).
Objetivou-se, neste trabalho avaliar a qualidade química, física e microbiológica de produtos minimamente processados comercializados em Lavras - MG, Brasília DF e São Paulo - SP.

\section{MATERIAL E MÉTODOS}

Um total de 144 amostras de produtos hortifruticolas minimamente processados, foram coletados em gôndolas de supermercados nas cidades de Lavras -MG, Brasília DF e São Paulo -SP, sendo 48 produtos por cidade. Em Lavras, foram coletadas melancia fatiada ao meio, moranga e abóbora (fracionada em quatro partes), salada de frutas, em dois supermercados; em Brasília, foram coletados (espinafre picado), alface crespa (fracionada), abóbora (cortada em cubos) e vagem (fatiada em rodelas), em três supermercados; e em São Paulo foram coletados couve (picada), abacaxi (descascado), moranga (cortada em cubos) e salada de alface (fracionada), em dois supermercados.

Para cada cidade foram realizadas três coletas a cada vinte dias, sendo coletado quatro produtos por cidade, com duas repetições. As avaliações de qualidades foram realizadas considerando-se dois períodos de análise: a fabricação e a validade dos produtos . As coletas foram realizadas na data de fabricação dos produtos cujas bandejas ou unidades experimentais continham 150 - 200 gramas de produto minimamente processado, eles foram coletados em gôndolas de supermercado no período da manhã e transportados em caixas isotérmicas, previamente higienizadas contendo gelox. Posteriormente, as amostras foram encaminhadas para análises nos Laboratórios de Microbiologia de Alimentos e de Fisiologia e Pós-Colheita de Frutos e Hortaliças do Departamento de Ciência dos Alimentos da Universidade Federal de Lavras.

Para os PMP coletados em Lavras, melancia, abóbora e moranga, a validade foi de sete dias e a salada de frutas, um dia. Em Brasília, todos os PMP coletados tiveram validade de sete dias. Em São Paulo, todos os PMP coletados apresentaram na embalagem validade de cinco dias.

As determinações químicas realizadas foram: $\mathrm{pH}$, acidez titulável (AT) e sólidos solúveis (SS) (AOAC, 1992). Pesquisas de coliformes a $45^{\circ} \mathrm{C}$, determinação de Escherichia coli (SILVA et al., 1997).

Foi realizada a análise de variância com o desdobramento das interações significativas e comparação de médias pelo teste Scott-Knot, a 5\% de probabilidade (TRIOLA, 2005).

$\mathrm{O}$ experimento foi conduzido em delineamento inteiramente casualizado (DIC) com arranjo fatorial $4 \times 3 \times 2$, 
sendo quatro produtos, três coletas (a cada 20 dias) e duas avaliações (fabricação e validade) em cada cidade, por coleta. A TABELA 1 apresenta as cidades, com as respectivas datas de coletas dos produtos minimamente processados.

\section{RESULTADOS E DISCUSSÃO}

A qualidade não é um atributo único bem definido e sim, um conjunto de muitas propriedades ou características peculiares de cada produto hortícola. Importantes fatores nessa qualidade são o $\mathrm{pH}$, juntamente com os teores de acidez titulável (AT) e de sólidos solúveis (SS), sendo responsáveis pelo sabor desses produtos através da sua acidez e doçura (CHITARRA \& CHITARRA, 2005).

Entre os produtos minimamente processados (melancia, abóbora, moranga e salada de frutas) coletados em Lavras, observaram-se diferenças significativas nos valores do $\mathrm{pH}$, em relação aos períodos de coletas, sendo que, na maioria desses produtos, os maiores valores dessa variável ocorreram na $3^{\mathrm{a}}$ coleta. Ainda em relação ao $\mathrm{pH}$, constataram-se diferenças entre a data de fabricação e validades na $3^{\text {a }}$ coleta, com exceção da salada de frutas, que se apresentou semelhante entre as datas nas coletas (TABELA 2).

A acidez titulável (AT) mostrou-se significativamente afetada pelos períodos de coletas, sendo que a melancia, abóbora e moranga comercializadas em Lavras apresentaram diferenças entre as coletas, em contrapartida a salada de frutas foi semelhante (TABELA 3).

Somente a salada de frutas comercializada em Lavras apresentou diferenças entre as coletas, no que diz respeito ao teor de sólidos solúveis (SS), com menor valor dessa variável na $3^{\mathrm{a}}$ coleta $(8,8 \%)$ (TABELA 4$)$. Pinto (2007), caracterizando a qualidade de produtos minimamente processados (melancia, abóbora, moranga e salada de frutas), comercializados em Lavras nas diferentes estações do ano, observou variações nos valores de pH, AT e SS no decorrer do ano, contudo, não verificou influência da fabricação e validade sobre as características de qualidade desses produtos.

Tabela 1 - Datas das coletas nas respectivas cidades avaliadas.

\begin{tabular}{cccc}
\hline Cidades & \multicolumn{3}{c}{ Coletas } \\
\hline & $1^{\mathrm{a}}$ & $2^{\mathrm{a}}$ & $3^{\mathrm{a}}$ \\
\hline Lavras - MG & $05 \backslash 10 \backslash 2004$ & $25 \backslash 10 \backslash 2004$ & $15 \backslash 11 \backslash 2004$ \\
Brasília - DF & $06 \backslash 12 \backslash 2004$ & $26 \backslash 12 \backslash 2004$ & $16 \backslash 01 \backslash 2005$ \\
São Paulo - SP & $10 \backslash 02 \backslash 2005$ & $02 \backslash 03 \backslash 2005$ & $22 \backslash 03 \backslash 2005$ \\
\hline
\end{tabular}

Tabela 2 - Valores de pH observados em melancia, abóbora, moranga, e salada de frutas em função da época de coleta e vida de prateleira para PMP, coletados na cidade de Lavras -MG. UFLA, Lavras, MG, 2005.

\begin{tabular}{|c|c|c|c|c|}
\hline & \multirow[t]{2}{*}{ Avaliações } & \multicolumn{3}{|c|}{ Coletas } \\
\hline & & $1^{\mathrm{a}}$ & $2^{a}$ & $3^{\mathrm{a}}$ \\
\hline \multirow[t]{2}{*}{ Melancia } & Fabricação & 5,3 a A & 5,4 a A & 5,5 a A \\
\hline & Validade & 5,5 a A & $5,5 \mathrm{a} \mathrm{A}$ & $6,0 \mathrm{~b} \mathrm{~B}$ \\
\hline \multirow[t]{2}{*}{ Abóbora } & Fabricação & $6,4 \mathrm{~b} \mathrm{~A}$ & $6,7 \mathrm{c} \mathrm{A}$ & 5,9 a A \\
\hline & Validade & 6,5 a A & 6,5 a A & $6,8 \mathrm{~b} \mathrm{~B}$ \\
\hline \multirow[t]{2}{*}{ Moranga } & Fabricação & 6,4 a $\mathrm{A}$ & 6,3 a $A$ & 6,4 a $A$ \\
\hline & Validade & 6,2 a $\mathrm{A}$ & 6,2 a A & $7,0 \mathrm{~b} \mathrm{~B}$ \\
\hline \multirow[t]{2}{*}{ Sal. frutas } & Fabricação & $4,2 \mathrm{~b} \mathrm{~A}$ & 4,0 a A & 4,0 a $\mathrm{A}$ \\
\hline & Validade & $4,2 \mathrm{~b} \mathrm{~A}$ & 3,9 a A & 3,9 a A \\
\hline
\end{tabular}

${ }^{11}$ Médias seguidas da mesma letra minúscula na linha não diferem significativamente entre si pelo teste de Scott-Knott, a 5\% de probabilidade.

${ }^{21}$ Médias seguidas da mesma letra maiúscula na coluna para cada produto não diferem significativamente entre si pelo teste de F, a $5 \%$ de probabilidade. 
Os produtos comercializados em Brasília (espinafre, vagem, abóbora e alface crespa) apresentaram variações nos valores de $\mathrm{pH}$ (TABELA 5), acidez titulável (AT) (TABELA 6) e sólidos solúveis (SS) (TABELA 7), entre os períodos de coletas e entre as datas de fabricação e validade, com exceção da alface crespa em relação ao pH e o espinafre nos teores de SS, que não apresentaram diferenças entre o processamento e o prazo final de validade.
A couve, em relação ao abacaxi, moranga e salada de frutas comercializados em São Paulo, apresentou variações entre as coletas, no que se refere ao $\mathrm{pH}$, onde o maior valor dessa variável para esse produto foi encontrado na $3^{\mathrm{a}}$ coleta, 6,8 (TABELA 8).

Com relação à $\mathrm{AT}$, o abacaxi e a couve, apresentaram diferenças significativas entre as

Tabela 3 - Teores de acidez titulável \% de ácido cítrico observados em PMP, coletados em Lavras - MG durante três coletas. UFLA, Lavras, MG, 2005.

\begin{tabular}{lccc}
\hline \multicolumn{1}{c}{ Produtos } & \multicolumn{1}{c}{ Coletas } \\
\cline { 2 - 4 } & $1^{\mathrm{a}}$ & $2^{\mathrm{a}}$ & $3^{\mathrm{a}}$ \\
\hline Melancia & $0,20 \mathrm{a}$ & $0,32 \mathrm{c}$ & $0,28 \mathrm{~b}$ \\
Abóbora & $0,07 \mathrm{a}$ & $0,12 \mathrm{~b}$ & $0,06 \mathrm{a}$ \\
Moranga & $0,11 \mathrm{a}$ & $0,17 \mathrm{~b}$ & $0,12 \mathrm{a}$ \\
Salada de frutas & $0,20 \mathrm{a}$ & $0,20 \mathrm{a}$ & $0,20 \mathrm{a}$ \\
\hline
\end{tabular}

${ }^{11}$ Médias seguidas da mesma letra na linha não diferem significativamente entre si pelo teste de Scott-Knott, a 5\% de probabilidade

Tabela 4 - Teores de sólidos solúveis (SS) (\%) observados em produtos coletados em Lavras - MG durante três coletas. UFLA, Lavras, MG, 2005.

\begin{tabular}{lccc}
\hline \multicolumn{1}{c}{ Produtos } & \multicolumn{2}{c}{ Coletas } & $3^{\mathrm{a}}$ \\
\cline { 2 - 4 } & \multicolumn{2}{c}{$1^{\mathrm{a}}$} & $9,8 \mathrm{a}$ \\
\hline Melancia MP & $9,8 \mathrm{a}$ & $9,7 \mathrm{a}$ & $2,9 \mathrm{a}$ \\
Abóbora MP & $3,2 \mathrm{a}$ & $2,9 \mathrm{a}$ & $3,8 \mathrm{a}$ \\
Moranga MP & $4,5 \mathrm{a}$ & $4,1 \mathrm{a}$ & $8,8 \mathrm{~b}$ \\
Salada de frutas & $13,7 \mathrm{a}$ & $13,7 \mathrm{a}$ & $2^{\mathrm{a}}$ \\
\hline
\end{tabular}

${ }^{11}$ Médias seguidas da mesma letra na linha não diferem significativamente entre si pelo teste de Scott-Knott, a 5\% de probabilidade

Tabela 5 - Valores de pH observados em espinafre, vagem, abóbora, e alface crespa em função da época de coleta e vida de prateleira para PMP, coletados na cidade de Brasília - DF. UFLA, Lavras, MG, 2005.

\begin{tabular}{|c|c|c|c|c|}
\hline \multirow{2}{*}{\multicolumn{2}{|c|}{ Avaliações }} & \multicolumn{3}{|c|}{ Coletas } \\
\hline & & \multirow{2}{*}{$\frac{1^{\mathrm{a}}}{6,4 \mathrm{a} \mathrm{A}}$} & \multirow{2}{*}{$\frac{2^{\mathrm{a}}}{7,1 \mathrm{~b} \mathrm{~A}}$} & \multirow{2}{*}{$\frac{3^{\mathrm{a}}}{8,2 \mathrm{cA}}$} \\
\hline Espinafre & Fabricação & & & \\
\hline & Validade & 6,6 a $A$ & 7,6 b B & 9,6 c B \\
\hline \multirow[t]{2}{*}{ Vagem } & Fabricação & 6,5 a $A$ & 6,4 a $A$ & 6,5 a $A$ \\
\hline & Validade & 6,4 a A & 7,1 b B & 6,3 a $A$ \\
\hline \multirow[t]{2}{*}{ Abóbora } & Fabricação & 6,7 a $A$ & 6,8 a $A$ & $7,7 \mathrm{~b} \mathrm{~A}$ \\
\hline & Validade & 6,8 a A & 7,7 b B & 8,2 c B \\
\hline \multirow[t]{2}{*}{ Alface C. } & Fabricação & 6,3 a $A$ & 6,7 a $A$ & $7,4 \mathrm{~b} \mathrm{~A}$ \\
\hline & Validade & 6,4 a $A$ & 6,7 a A & 7,6 b A \\
\hline
\end{tabular}

${ }^{11}$ Médias seguidas da mesma letra minúscula na linha não diferem significativamente entre si pelo teste de Scott-Knott, a 5\% de probabilidade.

${ }^{21}$ Médias seguidas da mesma letra maiúscula na coluna para cada produto não diferem significativamente entre si pelo teste de $\mathrm{F}$, a $5 \%$ de probabilidade

Ciênc. agrotec., Lavras, v. 33, n. 1, p. 219-227, jan./fev., 2009 
coletas. Já entre a data de fabricação e a validade, somente a salada de alface mostrou-se semelhante, sendo que os demais produtos apresentaram variações (TABELA 9).

Os valores de sólidos solúveis foram afetados significativamente pelo período de coleta. A couve e a salada de alface apresentaram variações, com maior valor dessa variável na $2^{\text {a }}$ coleta, 6,2 e 3,3\%, respectivamente (TABELA 10).

Os resultados obtidos no presente trabalho apontam a existência de desvios no padrão de qualidade dos produtos minimamente processados comercializados em Lavras - MG, Brasília - DF e São Paulo - SP. Resultados semelhantes foram encontrados por Pinto (2007) que pesquisou a qualidade de produtos hortifrutícolas minimamente processados, comercializados em Lavras nas estações do ano. Logo, alterações nas variáveis citadas, ao longo das coletas, indicaram possíveis alterações na qualidade sensorial dos alimentos, em especial na acidez e doçura. Não obstante, embora a busca por produtos minimamente processados com padrão de qualidade deva ser incessante, a obtenção desses produtos é de difícil êxito, considerando-se a dependência da qualidade e as condições edafo-climáticas as quais as culturas são submetidas, diferença entre cultivares e mesmo variações na montagem de saladas de frutas.

Tabela 6 - Teores de acidez titulável (\% de ácido cítrico) observado em espinafre, vagem, abóbora e alface crespa em função da época de coleta e vida de prateleira para PMP, coletados na cidade Brasília - DF. UFLA, Lavras, MG, 2005.

\begin{tabular}{|c|c|c|c|c|}
\hline \multirow{2}{*}{\multicolumn{2}{|c|}{ Avaliações }} & \multicolumn{3}{|c|}{ Coletas } \\
\hline & & $1^{\mathrm{a}}$ & $2^{a}$ & $3^{\mathrm{a}}$ \\
\hline \multirow[t]{2}{*}{ Espinafre } & Fabricação & 0,13 b B & $0,13 \mathrm{~b} \mathrm{~B}$ & 0,09 a B \\
\hline & Validade & $0,11 \mathrm{c} \mathrm{A}$ & $0,07 \mathrm{~b} A$ & 0,02 a $A$ \\
\hline \multirow[t]{2}{*}{ Vagem } & Fabricação & $0,12 \mathrm{~b} \mathrm{~A}$ & 0,11 a B & 0,20 c B \\
\hline & Validade & $0,12 \mathrm{c} \mathrm{A}$ & 0,09 b A & 0,08 a $\mathrm{A}$ \\
\hline \multirow[t]{2}{*}{ Abóbora } & Fabricação & $0,12 \mathrm{~b} \mathrm{~A}$ & 0,11 a B & $0,12 \mathrm{~b} \mathrm{~B}$ \\
\hline & Validade & 0,12 c A & $0,08 \mathrm{~b} \mathrm{~A}$ & 0,04 a A \\
\hline \multirow[t]{2}{*}{ Alface C. } & Fabricação & 0,17 c B & 0,07 a B & $0,09 \mathrm{~b} \mathrm{~B}$ \\
\hline & Validade & $0,08 \mathrm{c} \mathrm{A}$ & $0,06 \mathrm{~b} \mathrm{~A}$ & 0,04 a A \\
\hline
\end{tabular}

${ }^{11}$ Médias seguidas da mesma letra minúscula na linha não diferem significativamente entre si pelo teste de Scott-Knott, a $5 \%$ de probabilidade.

${ }^{21}$ Médias seguidas da mesma letra maiúscula na coluna para cada produto não diferem significativamente entre si pelo teste de $\mathrm{F}$, a $5 \%$ de probabilidade

Tabela 7 - Teores de sólidos solúveis (SS) (\%) observado em espinafre, vagem, abóbora e alface crespa em função da época de coleta e vida de prateleira para PMP, coletados em Brasília - DF. UFLA, Lavras, MG, 2005.

\begin{tabular}{|c|c|c|c|c|}
\hline & \multirow[t]{2}{*}{ Avaliações } & \multicolumn{3}{|c|}{ Coletas } \\
\hline & & $1^{\mathrm{a}}$ & $2^{a}$ & $3^{a}$ \\
\hline \multirow[t]{2}{*}{ Espinafre } & Fabricação & 3,3 a B & 3,0 a B & 3,0 a B \\
\hline & Validade & 2,7 a A & 2,3 a A & 2,0 a $\mathrm{A}$ \\
\hline \multirow[t]{2}{*}{ Vagem } & Fabricação & $3,5 \mathrm{~b} \mathrm{~B}$ & 4,6 a B & $3,0 \mathrm{~b} \mathrm{~A}$ \\
\hline & Validade & $2,7 \mathrm{~b} \mathrm{~A}$ & 3,5 a A & 3,3 a $A$ \\
\hline \multirow[t]{2}{*}{ Abóbora } & Fabricação & 3,9 c A & 7,8 a B & $5,2 \mathrm{~b} \mathrm{~A}$ \\
\hline & Validade & $5,4 \mathrm{c} \mathrm{B}$ & $6,0 \mathrm{~b} \mathrm{~A}$ & 7,5 a B \\
\hline \multirow[t]{2}{*}{ Alface crespa } & Fabricação & 3,7 a B & $2,0 \mathrm{~b} \mathrm{~A}$ & $2,2 \mathrm{~b} \mathrm{~A}$ \\
\hline & Validade & 2,7 a A & $1,6 \mathrm{~b} \mathrm{~A}$ & $2,0 \mathrm{~b} \mathrm{~A}$ \\
\hline
\end{tabular}


Tabela 8 - Valores de pH observado em PMP, coletados em São Paulo - SP, durante três coletas. UFLA, Lavras, MG, 2005.

\begin{tabular}{|c|c|c|c|}
\hline \multirow{2}{*}{ Produtos } & \multicolumn{3}{|c|}{ Coletas } \\
\hline & $1^{\mathrm{a}}$ & $2^{a}$ & $3^{a}$ \\
\hline Abacaxi & $3.8 \mathrm{a}$ & $4,1 \mathrm{a}$ & $4,1 \mathrm{a}$ \\
\hline Couve & $6,0 \mathrm{a}$ & $6,1 \mathrm{a}$ & $6,8 \mathrm{~b}$ \\
\hline Moranga & $5,8 \mathrm{a}$ & $6,0 \mathrm{a}$ & $6,0 \mathrm{a}$ \\
\hline S. Alface & $6,2 \mathrm{a}$ & $5,9 \mathrm{a}$ & $6,6 \mathrm{a}$ \\
\hline
\end{tabular}

"Médias seguidas da mesma letra na linha não diferem significativamente entre si pelo teste de Scott-Knott, a 5\% de probabilidade.

Tabela 9 - Teores de acidez titulável (\% de ácido cítrico) observado em abacaxi, couve, moranga e saladas de alfaces em função da época de coleta e vida de prateleira para PMP coletados na cidade São Paulo - SP. UFLA, Lavras, MG, 2005 .

\begin{tabular}{llccc}
\hline \multirow{2}{*}{ Avaliações } & \multicolumn{3}{c}{ Coletas } \\
\cline { 3 - 5 } Abacaxi & Fabricação & 0,7 a A & 0,7 a A & 0,9 b B \\
& Validade & 0,8 b B & 0,6 a A & 0,7 a A \\
\hline \multirow{2}{*}{ Couve } & Fabricação & 0,2 a A & 0,3 b A & 0,2 a B \\
& Validade & 0,2 b A & 0,3 c A & 0,1 a A \\
\hline \multirow{2}{*}{ Moranga } & Fabricação & 0,13 a B & 0,15 a B & 0,15 a B \\
& Validade & 0,10 a A & 0,10 a A & 0,10 a A \\
\hline \multirow{2}{*}{ S. Alface } & Fabricação & 0,06 a A & 0,08 a A & 0,09 a A \\
& Validade & 0,08 a A & 0,08 a A & 0,09 a A \\
\hline
\end{tabular}

${ }^{11}$ Médias seguidas da mesma letra minúscula na linha não diferem significativamente entre si pelo teste de Scott-Knott, a 5\% de probabilidade.

${ }^{2 \backslash}$ Médias seguidas da mesma letra maiúscula na coluna para cada produto não diferem significativamente entre si pelo teste de $\mathrm{F}$, a $5 \%$ de probabilidade

Tabela 10 - Teores de sólidos solúveis (SS) (\%) observados em produtos coletados em São Paulo - SP durante três coletas. UFLA, Lavras, MG, 2005.

\begin{tabular}{lccc}
\hline \multicolumn{1}{c}{ Produtos } & \multicolumn{3}{c}{ Coletas } \\
\cline { 2 - 4 } & $1^{\mathrm{a}}$ & $2^{\mathrm{a}}$ & $3^{\mathrm{a}}$ \\
\hline Abacaxi MP & $11,1 \mathrm{a}$ & $11,1 \mathrm{a}$ & $11,5 \mathrm{a}$ \\
Couve MP & $3,5 \mathrm{a}$ & $6,2 \mathrm{~b}$ & $3,3 \mathrm{a}$ \\
Moranga MP & $4,0 \mathrm{a}$ & $4,9 \mathrm{a}$ & $4,4 \mathrm{a}$ \\
S. Alface & $2,3 \mathrm{a}$ & $3,3 \mathrm{~b}$ & $2,0 \mathrm{a}$ \\
\hline
\end{tabular}

${ }^{1 "}$ Médias seguidas da mesma letra na linha não diferem significativamente entre si pelo teste de Scott-Knott, a 5\% de probabilidade

Foi observado interação significativa entre produtos, épocas de coletas e vida de prateleira na cidade Lavras (melancia, abóbora, moranga e salada de frutas), Brasília (espinafre, vagem, abóbora e alface crespa) e São Paulo (abacaxi, couve, moranga e salada de alface) para a variável coliformes a $45^{\circ} \mathrm{C}$. A Agência Nacional de Vigilância Sanitária (ANVISA) pela Resolução RDC n ${ }^{\circ} 12$ de 02 de janeiro de 2001, estabelece limite máximo de $5 \times 10^{2} \mathrm{NMP} / \mathrm{g}$ (2,7 ciclos log) para coliformes a $45^{\circ} \mathrm{C}$ e a ausência de Salmonella, em $25 \mathrm{~g}$ do produto (BRASIL, 2005). 
Em Lavras, os produtos melancia, abóbora, moranga e a salada de frutas apresentaram acima dos limites microbiológicos de 2,7 ciclos log para coliformes a $45^{\circ} \mathrm{C}$, estabelecidos pela RDC n ${ }^{\circ} 12$ da ANVISA (BRASIL, 2005) considerando o período entre a fabricação e o prazo de validade dos hortifrutícolas minimamente processados estabelecido pelos fabricantes (TABELA 11).

Da mesma forma, foi detectado nos hortifruticolas minimamente processados coletados em Brasília e São Paulo, falhas no padrão de qualidade quanto aos índices de contaminação por coliformes a $45^{\circ} \mathrm{C}$, entre a data de processamento e o prazo de validade. Em Brasília, essa contaminação foi extremamente abusiva tanto na fabricação quanto na validade dos produtos. Ambos os produtos não atenderam aos padrões microbiológicos recomendados pela Legislação Federal. (TABELA 12). Dados compatíveis foram encontrados por Rosa (2002), que analisando salsão e broto de alfafa detectou contagens ultrapassando o valor máximo para coliformes a $45^{\circ} \mathrm{C}$, tanto para os padrões nacionais como internacionais. Em São Paulo, as contaminações foram maiores para couve e moranga e salada de alface (TABELA 13). A utilização de águas poluídas, esterco animal ou sedimentos de dejetos são citados como as principais fontes desse patógenos nesses produtos (GAGLIARDI \& KARNS, 2000).

Tabela 11 - Contagens de coliformes a $45^{\circ} \mathrm{C}(\log \mathrm{NMP} / \mathrm{g})$ observado em melancia, abóbora, moranga e salada de frutas em função da época de coleta e vida de prateleira para PMP coletados em Lavras - MG. UFLA, Lavras, MG, 2005.

\begin{tabular}{clccc}
\hline \multirow{2}{*}{ Avaliações } & \multicolumn{3}{c}{ Coletas } \\
\cline { 2 - 5 } Melancia & Fabricação & $0,5 \mathrm{a} \mathrm{A}$ & $0,5 \mathrm{a} \mathrm{A}$ & $0,9 \mathrm{a} \mathrm{A}$ \\
& Validade & $0,8 \mathrm{a} \mathrm{A}$ & $3,8 \mathrm{~b} \mathrm{~B}$ & $0,8 \mathrm{a} \mathrm{A}$ \\
\hline \multirow{2}{*}{ Abóbora } & Fabricação & $0,5 \mathrm{a} \mathrm{A}$ & $0,5 \mathrm{a} \mathrm{A}$ & $0,7 \mathrm{a} \mathrm{A}$ \\
& Validade & $0,5 \mathrm{a} \mathrm{A}$ & $5,0 \mathrm{~b} \mathrm{~B}$ & $1,1 \mathrm{a} \mathrm{A}$ \\
\hline \multirow{2}{*}{ Moranga } & Fabricação & $0,5 \mathrm{a} \mathrm{A}$ & $0,5 \mathrm{a} \mathrm{A}$ & $3,4 \mathrm{~b} \mathrm{~B}$ \\
& Validade & $3,0 \mathrm{~b} \mathrm{~B}$ & $5,0 \mathrm{c} \mathrm{B}$ & $1,0 \mathrm{a} \mathrm{A}$ \\
\hline \multirow{2}{*}{ Sal. frutasa } & Fabricação & $2,0 \mathrm{~b} \mathrm{~A}$ & $0,5 \mathrm{a} \mathrm{A}$ & $5,4 \mathrm{c} \mathrm{A}$ \\
& Validade & $3,4 \mathrm{a} \mathrm{B}$ & $4,2 \mathrm{~b} \mathrm{~B}$ & $5,4 \mathrm{c} \mathrm{A}$ \\
\hline
\end{tabular}

${ }^{11}$ Médias seguidas da mesma letra minúscula na linha não diferem significativamente entre si pelo teste de Scott-Knott, a 5\% de probabilidade.

${ }^{21}$ Médias seguidas da mesma letra maiúscula na coluna para cada produto não diferem significativamente entre si pelo teste de $\mathrm{F}$, a $5 \%$ de probabilidade

Tabela 12 - Contagens de coliformes a $45^{\circ} \mathrm{C}(\log \mathrm{NMP} / \mathrm{g})$ observado em espinafre, vagem, abóbora e alface crespa em função da época de coleta e vida de prateleira para PMP coletados em Brasília - DF. UFLA, Lavras, MG, 2005.

\begin{tabular}{llccc}
\hline \multirow{2}{*}{ Avaliações } & \multicolumn{3}{c}{ Coletas } \\
\cline { 3 - 5 } & & $1^{\mathrm{a}}$ & $2^{\mathrm{a}}$ & $3^{\mathrm{a}}$ \\
\hline \multirow{2}{*}{ Espinafre } & Fabricação & $6,0 \mathrm{~b} \mathrm{~A}$ & $4,3 \mathrm{a} \mathrm{A}$ & $6,3 \mathrm{~b} \mathrm{~A}$ \\
& Validade & $7,0 \mathrm{c} \mathrm{B}$ & $5,2 \mathrm{a} \mathrm{B}$ & $6,2 \mathrm{~b} \mathrm{~A}$ \\
\hline \multirow{2}{*}{ Vagem } & Fabricação & $6,1 \mathrm{~b} \mathrm{~A}$ & $5,0 \mathrm{a} \mathrm{A}$ & $6,3 \mathrm{~b} \mathrm{~A}$ \\
& Validade & $7,0 \mathrm{~b} \mathrm{~B}$ & $6,0 \mathrm{a} \mathrm{B}$ & $7,9 \mathrm{c} \mathrm{B}$ \\
\hline \multirow{2}{*}{ Abóbora } & Fabricação & $6,0 \mathrm{~b} \mathrm{~A}$ & $4,2 \mathrm{a} \mathrm{A}$ & $6,7 \mathrm{c} \mathrm{A}$ \\
& Validade & $7,0 \mathrm{~b} \mathrm{~B}$ & $5,3 \mathrm{a} \mathrm{B}$ & $7,9 \mathrm{c} \mathrm{B}$ \\
\hline \multirow{2}{*}{ Alface C. } & Fabricação & $4,6 \mathrm{c} \mathrm{A}$ & $0,6 \mathrm{a} \mathrm{A}$ & $1,5 \mathrm{~b} \mathrm{~A}$ \\
& Validade & $4,7 \mathrm{c} \mathrm{A}$ & $0,8 \mathrm{a} \mathrm{A}$ & $3,5 \mathrm{~b} \mathrm{~B}$ \\
\hline
\end{tabular}

${ }^{11}$ Médias seguidas da mesma letra minúscula na linha não diferem significativamente entre si pelo teste de Scott-Knott, a 5\% de probabilidade.

${ }^{2 \uparrow}$ Médias seguidas da mesma letra maiúscula na coluna para cada produto não diferem significativamente entre si pelo teste de $\mathrm{F}$, a $5 \%$ de probabilidade 
Tabela 13 - Contagens de coliformes a $45^{\circ} \mathrm{C}(\log \mathrm{NMP} / \mathrm{g})$ observado em abacaxi, couve, moranga e salada de alface em função da época de coleta e vida de prateleira para PMP, coletados em São Paulo - SP. UFLA, Lavras, MG, 2005.

\begin{tabular}{|c|c|c|c|c|}
\hline \multirow{2}{*}{\multicolumn{2}{|c|}{ Avaliações }} & \multicolumn{3}{|c|}{ Coletas } \\
\hline & & $1^{\mathrm{a}}$ & $2^{\mathrm{a}}$ & $3^{\mathrm{a}}$ \\
\hline \multirow[t]{2}{*}{ Abacaxi } & Fabricação & 0,3 a $\mathrm{A}$ & 0,3 a $A$ & $2,4 \mathrm{~b} \mathrm{~A}$ \\
\hline & Validade & 0,6 a A & 0,3 a $\mathrm{A}$ & $4,5 \mathrm{~b} \mathrm{~B}$ \\
\hline \multirow[t]{2}{*}{ Couve } & Fabricação & 6,0 с A & 4,3 a $A$ & $5,2 \mathrm{~b} \mathrm{~A}$ \\
\hline & Validade & 5,7 a $\mathrm{A}$ & 5,4 a B & $6,5 \mathrm{~b} \mathrm{~B}$ \\
\hline \multirow[t]{2}{*}{ Moranga } & Fabricação & 6,0 b B & 4,3 a $A$ & 4,3 a $\mathrm{A}$ \\
\hline & Validade & 4,7 a A & $5,3 \mathrm{~b} \mathrm{~B}$ & 4,4 a $\mathrm{A}$ \\
\hline \multirow[t]{2}{*}{ S. alface } & Fabricação & 5,3 c B & 2,5 a B & $3,3 \mathrm{~b} \mathrm{~A}$ \\
\hline & Validade & 1,3 a $\mathrm{A}$ & 1,0 a A & $3,4 \mathrm{~b} \mathrm{~A}$ \\
\hline
\end{tabular}

${ }^{11}$ Médias seguidas da mesma letra minúscula na linha não diferem significativamente entre si pelo teste de Scott-Knott, a 5\% de probabilidade.

${ }^{21}$ Médias seguidas da mesma letra maiúscula na coluna para cada produto não diferem significativamente entre si pelo teste de $\mathrm{F}$, a $5 \%$ de probabilidade.

$\mathrm{O}$ índice de coliforme a $45^{\circ} \mathrm{C}$ é empregado como indicador de contaminação fecal, visto presumir-se que a população desse grupo é constituída apenas de E. coli, que tem seu hábitat exclusivamente no trato intestinal. Sua presença indica a possibilidade de ocorrência de outros enteropatógenos, como Salmonella e Shigella (FRANCO \& LANDGRAF, 2005).

Os produtos moranga fatiada, saladas de frutas (Lavras), espinafre, vagem, abóbora japonesa (Brasília), couve e moranga cortada (São Paulo) apresentaram contaminação proveniente da matéria-prima ou manipulação pela enterobactéria $E$. coli, na data de fabricação. A contaminação de alimentos por alguns membros do grupo coliformes, particularmente produtos minimamente processados oferecidos prontos para o consumo, como Escherichia, Citrobacter, Enterobacter e Klebsiella, ou por espécies de Salmonella e Shigella, podem ser a causa de severas toxinfecções (SILVA et al., 2007).

Por isso elas são consideradas como bons indicadores de práticas de higiene e processos de produção inadequados. Gleeson \& O'Beirne (2005) verificou que durante o processamento ocorre a liberação de fluídos intracelulares, facilitando a multiplicação dos microrganismos. Contudo, não foi detectada a presença de Salmonella sp. nos produtos MP comercializados em Lavras, Brasília e São Paulo.

\section{CONCLUSÕES}

Os resultados mostraram falhas na manutenção da qualidade dos produtos hortifrutícolas minimamente processados, ao longo do seu prazo de validade, em supermercados de Lavras, com base na acidez titulavel e sólidos solúveis e em Brasília com base no $\mathrm{pH}$, acidez titulavel e sólidos solúveis e de São Paulo, com base no pH e sólidos solúveis.

Os consumidores dos três mercados estudados, Lavras - MG, Brasília - DF e São Paulo - SP estiveram expostos ao risco de ingestão de microrganismos patogênicos e ou deterioradores em diferentes épocas de coletas. Essas avaliações apontaram falhas quanto as Boas Práticas Fabricação (BPF), dos fornecedores de hortifruticolas minimamente processados avaliados.

\section{REFERÊNCIAS BIBLIOGRÁFICAS}

ANDRADE, N.; BASTOS, M. S. R.; ANTUNES, M. A. Higiene e sanitização de frutas e hortaliças minimamente processadas. In: MORETTI, C. L. (Ed.). Processamento mínimo de frutas e hortaliças. Brasília, DF: Embrapa Informação Tecnológica, 2004.

ASSOCIATION OF OFFICIAL ANALYTICAL CHEMISTRY. Official methods of analysis of the Association of Official Analytical Chemistry. 12. ed. Washington, DC, 1992. $2 \mathrm{v}$.

BERBARI, S. A. G.; PASCHOALINO, J. E.; SILVEIRA, N. F. A. Efeito do cloro na água de lavagem para desinfecção de alface minimamente processada. Ciência e Tecnologia de Alimentos, Campinas, v. 21, n. 2, p. 197-201, maio/ago. 2001. 
BEUCHAT, L. R. Ecological factor influencing survival and growth of humans pathogens on raw fruits and vegetables. Microbes and Infections, v. 4, p. 413-423, 2002.

BRASIL. Ministério da Saúde. Agência Nacional de Vigilância Sanitária. Resolução RDC, de 12 de janeiro de

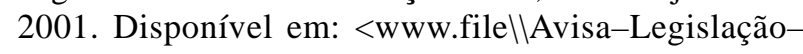
'Resolução.htm' Acesso em: 22 mar. 2005 .

CANTWELL, M. I.; SUSLOW, T. V. Postharvest handling systems: fresh cut fruits and vegetables. In: KADER, A. A. (Ed.). Postharvest technology of horticultural crops. 3. ed. Davis: Califórnia, 2002. cap. 36, p. 445-463.

CHITARRA, M. I. F.; CHITARRA, A. B. Pós-colheita de frutos e hortaliças: fisiologia e manuseio. 2. ed. Lavras: UFLA, 2005. $785 \mathrm{p}$.

DELIZA, R. Importância da qualidade sensorial em produtos minimamente processados. In: ENCONTRO NACIONAL SOBRE PROCESSAMENTO MÍNIMO DE FRUTAS E HORTALIÇAS, 2., 2000, Viçosa. Palestras... Viçosa: UFV, 2000.

FANTUSI, E.; PUSCHMANN, R.; VANETTI, M. C. D. Microbiota contaminante em repolho minimamente processado. Ciência e Tecnologia de Alimentos, Campinas, v. 24, n. 2, p. 207-211, abr./jun. 2004.

FRANCO, B. D. G. de M.; LANDGRAF, M. Microbiologia dos alimentos. São Paulo: Atheneu, 2005. 182 p.

GANGLIARDI, J. V.; KARNS, J. S. Leaching of Escherichia coli 0157: H7 in diverse soils under various agricultural management practices. Applied and Environmental Microbiology, Washington, v. 66, n. 3, p. 877-883, Mar. 2000.

GLESSON, E.; O'BEIRNE, D. Effects of process severity on surviral and growth of Escherichia coli and Listeria innocua on minimally processed vegetables. Food Control, v. 16, p. 677-685, 2005.
IFPA. International fresh-cut produce association. Disponível em: 〈http://www.fresh-cuts.org $\lambda$. Acesso em: 29 mar. 2005.

PINTO, D. M. Qualidade de produtos minimamente processados comercializados em diferentes épocas do ano. 2007. 116 p. Dissertação (Mestrado em Ciência dos Alimentos) - Universidade Federal de Lavras, Lavras, 2007.

ROSA, O. O. Microbiota associada a produtos hortícolas minimamente processados comercializados em supermercados. 2002. 202 p. Tese (Doutorado em Ciência dos Alimentos) - Universidade Federal de Lavras, Lavras, 2002.

ROSA, O. O.; CARVALHO, E. P. Características microbiológicas de frutos e hortaliças minimamente processadas. Boletim SBCTA, Campinas, v. 34, n. 2, p. 84$92,2000$.

SILVA JUNIOR, E. A. Manual de controle higiênicosanitário de alimentos. 4. ed. São Paulo: Varela, 2001. 477 p.

SILVA, N. da; JUNQUIRA, V. C. A.; SILVEIRA, N. F. A. Manual de métodos de análise microbiológica de alimentos. São Paulo: Varela, 1997. 295 p.

SILVA, N. da; JUNQUIRA, V. C. A.; SILVEIRA, N. F. A.; TANIWAKI, M. H.; SANTOS, R. F. S. dos; GOMES, R. A. $\mathrm{R}$. Manual de métodos de análise microbiológica de alimentos. 3. ed. São Paulo: Varela, 2007. 552 p.

TRIOLA, M. F. Introdução à estatística. 9. ed. Rio de Janeiro: LTC, 2005. $656 \mathrm{p}$.

VANETTI, M. C. D. Segurança microbiológica em produtos minimamente processados. In: ENCONTRO NACIONAL SOBRE PROCESSAMENTO MINIMO DE FRUTAS E HORTALIÇAS, 2004, Viçosa, MG. Anais... Viçosa: UFV, 2004. p. 30-31. 